\title{
La complejidad de los procesos educativos en el aula de clases
}

\section{A complexidade dos processos educacionais em sala de aula}

\section{Complexity of educational processes in the classroom}

\author{
Dr. Ariel Quezada* \\ Dr. Enrique Canessa ${ }^{* *}$
}

\begin{abstract}
RESUMEN
Dentro de un aula de clases se aloja una serie de procesos tanto individuales como sociales los cuales están integrados dentro de otro de mayor envergadura: educación. Al analizar un aula y las dinámicas que allí ocurren, sin lugar a dudas se está frente a un sistema que, como tal, está compuesto por individuos que se influyen entre si, emergiendo dinámicas que no se pueden explicar tan sólo por los comportamientos aislados de los individuos. Es así que, al hablar de sistemas en el comportamiento humano, tal como ocurre dentro de un aula, emerge naturalmente la vinculación con los sistemas complejos, es decir, aquellos que se componen de unidades interactuantes y excitables, de cuya acción en el tiempo emergen comportamientos no lineales, de los cuales la predictibilidad exacta es imposible. Esta nueva comprensión de los fenómenos en la naturaleza ha aglutinado a distintas disciplinas bajo un alero común que ha ayudado a encontrar importantes lazos de unión. El presente artículo tiene como objetivo entregar elementos que permiten comprender a los fenómenos educativos de aula como comportamientos propios de sistemas complejos. Asimismo, se presentará una herramienta que hace factible modelar el comportamiento dentro de la sala de clases, simulando su complejidad inherente.

* Universidad Adolfo Ibáñez, Viña del Mar Chile. Escuela de Psicología. Correio eletrônico:

** Facultad de Ingeniería y Ciencias.
\end{abstract} ariel.quezada@uai.cl 
Palabras-clave: Educación; sala de clases; sistemas complejos; no linealidad; modelamiento basado en agentes.

\title{
RESUMO
}

Dentro de uma sala de acolhimento de uma série de processos individuais e sociais que estão integradas no âmbito mais vasto da educação. Ao se analisar uma sala de aula e as dinâmicas que ocorrem lá, sem dúvida está a enfrentar um sistema que, como tal, é composto por indivíduos que estão influenciando-se mutuamente, dinâmicas emergentes que não pode ser explicada somente pelo comportamento dos isolados indivíduos. Então, ao falar de sistemas em comportamento humano, tais como ocorre dentro de uma sala de aula, emerge naturalmente a ligação a sistemas complexos, ou seja, aqueles que são compostas por unidades interactuantes e excitáveis, de cuja acção, no tempo emergem comportamentos não lineares, aos quais a previsibilidade é impossível. Esta nova compreensão dos fenómenos da natureza tem aglutinado diferentes disciplinas sob um conjunto comum que tem ajudado a encontrar links importantes. Este artigo tem como objetivo oferecer elementos para a compreensão dos fenômenos de sala de aula comportamentos próprios de sistemas complexos. Também se apresentará como uma ferramenta que torna viável modelar o comportamento dentro da sala de aula, simulando sua complexidade inerente.

Palavras-chave: Educação; sala de aula; sistemas complexos; não-linearidade; modelagem baseada agentes.

\begin{abstract}
Inside a classroom there are many individual as well as social processes, which are integrated into a bigger one: education. When one analyzes a classroom and the dynamics that exists inside it, there is no doubt that one is in presence of a system, which is composed of individuals who influence each other, and from that interactions it emerges dynamics that cannot be explained only in terms of the isolated behaviour of each individual. Hence, when speaking of human behaviour systems, as it happens in a classroom, it naturally emerges a link to complex systems, i.e. those systems which are composed of interacting and excitable parts. This interaction creates non-linear behaviour, which is not exactly predictable. This new understanding of natural phenomena has merged many different disciplines into a coherent frame, which has helped to find important common ground among them. The present paper aims at providing the elements which allow us to
\end{abstract}


understand the educational phenomena in the classroom as characteristic behaviour of complex systems. At the same time, the paper introduces a tool which makes possible to model the behaviour inside a classroom, including its inherent complexity.

Keywords: Education; classroom; complex systems; non-linear; agentbased modelling.

\section{Introducción}

Imagine a un profesor que tiene a cargo una asignatura cuyo objetivo es que los alumnos aprendan determinados contenidos. Recordando a Aristóteles, este profesor imagina a sus alumnos como tabulas rasas a las cuales se les debe grabar apropiadamente la información que pretende entregar. Para lograr este objetivo selecciona depuradamente los contenidos atingentes y, para asegurarse de transmitirlos bien, controla que no haya perturbaciones ni interrupciones. No obstante percibe que los resultados de aprendizaje no son los esperados. A su vez, el profesor trata de ocupar las calificaciones para motivar a los alumnos a que aprendan más, pero igualmente el resultado no es satisfactorio. Posteriormente ha sabido que si tiene alumnos motivados intrínsecamente los resultados podrían mejorar, por lo que los anima en términos de lograr un desafío personal y les hace ver que la educación será valiosa tanto para ellos como para su comunidad; sin embargo, los resultados aún no logran ser los esperados.

Siguiendo con el ejemplo del profesor, además de sus estrategias para mejorar los rendimientos de los alumnos, él constata que comúnmente existen alumnos altamente motivados y otros con escasa motivación, con frecuencia agrupados espontáneamente y muchas veces polarizándose en cada categoría: alumnos que les va bien y les sigue yendo bien o mejor, y otros que en un comienzo les va mal y siguen mal o empeoran. Además, constata que estos rendimientos no necesariamente están directamente relacionados con competencias o habilidades individuales de los alumnos.

Entonces, si el profesor se ha esforzado en aplicar diversas estrategias para mejorar el rendimiento de todos sus alumnos y aún así no observa una mejoría global y pareciera que se ha exacerbado el disímil rendimiento entre alumnos, ¿Qué estará ocurriendo?

Probablemente la primera estrategia del profesor está basada en una mirada del proceso educativo como si fuera una expresión de la Teoría de la Información 
(SHANNON, 1948), en la cual hay un emisor, un receptor, un canal de comunicación y un mensaje que se procura entregar evitando al máximo cualquier interferencia o ruido. Por lo tanto, la expectativa de éxito se basó en transmitir los contenidos de la manera más eficiente, reduciendo al máximo cualquier perturbación. En segunda instancia, el profesor se da cuenta que los alumnos no son meros receptores sino que ellos mismos desean recibir o no la información entregada por el profesor y aprenderla, por lo que integra otra variable más que es la motivación, tanto en sus variantes extrínseca (evaluaciones) e intrínseca (valoración individual y colectiva del conocimiento).

En las estrategias del profesor se puede observar la bien difundida tendencia a aplicar un paradigma lineal de causa-efecto para tratar de entender los procesos educativos. En esa lógica, hay un sistema simple al cual se quiere entregar la mayor cantidad de información sin pérdida de esta, a lo que posteriormente se agrega la variable motivacional, entre muchas variables que el educador podría intentar integrar para tener mejor expectativa de éxito. Así entra en juego otra tradición relacionada con tratar de incorporar la mayor cantidad de variables y controlarlas al máximo para lograr los objetivos educacionales propuestos. Estas estrategias corresponden a un paradigma que trata de explicar un sistema integrando el máximo de parámetros de observación, y que pretende controlarlos para así llegar a los resultados planificados. Sin embargo, no logra dar cuenta de la complejidad intrínseca del sistema, es decir, esta explicación intenta complicar un sistema desde una mirada simple, pero sin comprender toda su complejidad inherente.

En la mirada anterior falta reconocer que la complejidad de un sistema no está ligada necesariamente a la cantidad de variables que intervienen en él, sino que está asociada a la existencia de no linealidades, interacciones y múltiples lazos de realimentación entre las variables. Es cierto que para describir adecuadamente a un sistema debemos considerar la mayoría de sus variables; pero también es muy importante tener en cuenta la forma en que dichas variables se relacionan entre si. Esto podría ser incluso más fundamental que agregar más variables para tratar de entender el sistema. La complejidad del fenómeno no está tan solo en la gran cantidad de variables que intervienen, sino que también en la forma en que estas interactúan entre si.

En el ejemplo propuesto se puede observar que variadas experiencias educativas cotidianas pueden envolver una gran complejidad, la cual se puede entender mejor a través de un cambio de paradigma ligado a los Sistemas Complejos, el cual se analizará a continuación. 


\section{Sistemas complejos y sus propriedades}

Uno de los paradigmas de reciente creación es el de los Sistemas Complejos (SC). En general, un SC está compuesto por múltiples elementos individuales interactuantes y excitables entre si (agentes). Estos agentes cambian sus estados internos según la interacción que tienen con otros agentes y el ambiente en que están inmersos. Aunque el comportamiento individual de dichos agentes resulte ser simple, la interacción de estos produce comportamientos y características del sistema que como un todo son diferentes a una agregación lineal de sus conductas individuales (HOLLAND; MILLER, 1991). Aún más, el comportamiento colectivo puede ser una propiedad emergente del sistema, la que no se entiende adecuadamente si la tratamos como una propiedad del sistema mismo y no como algo que emerge de la interacción entre los agentes del sistema. Un simple y clásico ejemplo de lo anterior es una bandada de pájaros volando. A simple vista y mirando una bandada de pájaros en vuelo, el observador podría pensar que las aves se coordinan muy estrechamente y con un objetivo común compartido entre si para girar, subir, bajar, mantener y/o cambiar la dirección de vuelo. Sin embargo, está demostrado que este comportamiento inteligente es una propiedad emergente del sistema (bandada), basado en un comportamiento mucho más simple de cada pájaro (agente). En realidad, lo que hace cada ave es nada más que conservar una cierta distancia con los pájaros que lo rodean. Así, de un comportamiento individual muy sencillo, emerge un comportamiento grupal mucho más complejo. Al mismo tiempo, se observa otra característica propia de un SC: la autoorganización, es decir, de la interacción de sus agentes, sin conciencia ni planificación alguna, se genera orden espontáneo, representado en patrones espaciales y/o temporales en la dinámica del sistema.

Un ejemplo más próximo a la situación del aula se encuentra en el modelo de segregación social de Schelling (1989). Este es un modelo muy simple, en el cual existen agentes que se ubican sobre un tablero inicialmente en forma aleatoria. Los agentes son de dos tipos, por ejemplo, azules y rojos. Los agentes tienen preferencia por estar ubicados en zonas donde haya una cierta cantidad de agentes de su mismo color. Si esa condición no se cumple, estos cambian de lugar aleatoriamente. Este modelo tan simple demuestra que normalmente los agentes terminarán agrupándose en conglomerados de un mismo color, al igual como podría ocurrir en una sala de clases, en la cual los alumnos también podrían agruparse en conglomerados de alto y bajo desempeño, dependiendo de su tolerancia para aceptar trabajar con estudiantes de distinto rendimiento. 
Esta dinámica en un SC se aleja de un comportamiento lineal, es decir, aquel en el cual se puede realizar una predicción exacta de la conducta del sistema a partir del conocimiento de las condiciones iniciales de dicho sistema. En cambio, en un sistema con dinámica no lineal, una pequeña variación en las condiciones iniciales puede generar un comportamiento totalmente diferente a lo largo del tiempo. Volviendo al ejemplo inicial, si el sistema educativo fuera lineal y dependiera de una buena transmisión de información y motivación del alumnado, entonces dado un cierto nivel de dichas variables, se debería poder predecir el rendimiento de los alumnos. Sin embargo, se vio que el curso podría polarizarse, lo que indicaría una dinámica no lineal. Además, bajo esta condición, sería muy difícil predecir el comportamiento futuro del curso, como por ejemplo, pronosticar cuántos grupos de diferentes rendimientos habrá al finalizar el curso, dada la proporción inicial de alumnos con buen y mal rendimiento.

Lo interesante de establecer estas propiedades de un SC, es que se manifiestan en sistemas de muy diferente materialidad. Por ejemplo, en física, existe el modelo Ising de dipolos magnéticos (conjunto de moléculas que se comportan como microscópicos imanes) que en algunos materiales tienden a alinearse y crear fuerzas magnéticas que modifican las propiedades de los mismos (GLAUBER, 1963). Este fenómeno del mundo inerte exhibe características similares a lo que sucede en organizaciones y la forma en que se crean sus objetivos corporativos (COHEN; MARCH; OLSEN, 1972). Los miembros con objetivos personales similares tienden a tratar de alinear los objetivos organizacionales con dichos objetivos personales, modificando los objetivos corporativos y afectando las características de la organización. Además, dada la disímil materialidad de los dos ejemplos, se puede pensar que el paradigma de SC podría aglutinar diferentes modelos bajo uno solo, que permita entender unificada y holísticamente a los fenómenos que cada modelo trata de explicar por separado. Esta visión permitiría una mejor comprensión global de distintos fenómenos y es precisamente el espíritu que anima a estudiarlos interdisciplinariamente (NICOLIS; NICOLIS, 2007; SOLÉ; MANRUBIA, 2001).

Una vez esbozados los aspectos más relevantes del paradigma de SC, se procederá a analizar en detalle como estos se hacen presentes dentro de las aulas de clases, tanto a nivel individual como colectivo. 


\section{El aula mirada desde los sistemas complejos y sus implicancias}

Volviendo a la situación con que comenzamos este artículo, recordemos que el profesor observa con inquietud que los alumnos de su curso parecen polarizarse en dos grupos: aquellos con un adecuado rendimiento y otros con uno deficiente. Bajo la mirada lineal, esto no tiene una explicación simple, pues sería esperable un espectro amplio de rendimientos, acorde a las capacidades de cada alumno. Sin embargo, bajo la perspectiva de los SC, esta polarización se explica porque los alumnos buenos estarían inmersos en un círculo virtuoso (bucle con realimentación positiva incrementante), mientras que los alumnos con un rendimiento pobre serían parte de un círculo vicioso (bucle con realimentación positiva decreciente). En efecto, aquellos alumnos que comienzan el curso con una predisposición positiva y que rinden bien, se motivarán adecuadamente, aprenderán los contenidos y eso les permitirá estar mejor preparados para seguir bien el resto del curso. Por otra parte, aquellos alumnos que comienzan mal, estarán peor preparados para continuar con el aprendizaje de los contenidos del resto del curso. Al mismo tiempo, este ejemplo sugiere que este sistema educativo se autoorganiza, sin control ni intervención externa.

También, se debe considerar que el fenómeno de aprendizaje es una propiedad emergente tanto a nivel individual (alumno) como a nivel colectivo (conjunto de alumnos del curso). En el plano individual, sabemos que las neuronas cerebrales, fuente de nuestras capacidades cognitivas e intelectuales, son dispositivos muy simples. Se limitan a ponderar ciertas entradas (estímulos), luego estas entradas ponderadas se suman y si dicha suma excede un umbral, se gatilla una salida que es un estímulo para una entrada de otra neurona (Rumelhart, McClelland y PDP research group, 1986). Pareciera difícil pensar que un dispositivo (agente) tan sencillo fuera capaz de realizar alguna función superior o que tenga características interesantes (aprendizaje). Sin embargo, de la interacción de miles de millones de neuronas emergen propiedades tales como aprendizaje e inteligencia. Nótese que estas propiedades no son inherentes a una neurona en particular, sino que a la interconexión de un conjunto de muchas de estas. De hecho, en las ciencias de la computación existen dispositivos llamados redes neuronales artificiales (Artificial Neural Networks, ANN por su sigla en inglés). Las ANN están constituidas por neuronas artificiales que individualmente hacen lo mismo que una neurona cerebral, siendo capaces de realizar tareas sofisticadas tales como reconocimiento de patrones, clasificación y otras. Luego, debemos reconocer que el aprendizaje en un individuo se basa en estas propiedades emergentes de sus neuronas cerebrales. 
En una escala superior a la neuronal, es decir, a nivel de funcionamiento cognitivo, también se han encontrado evidencias de características propias de los sistemas complejos. Al realizar inferencias destinadas a la solución de problemas de localización espacial, se presentan patrones fractales en las estrategias de cada persona. La geometría fractal (aquella que describe figuras fractales) es la geometría propia de los sistemas complejos y describe distintas formas naturales a partir de su patrón de irregularidad, el cual se convierte en una pauta característica que se repite tanto en pequeñas como en grandes escalas de análisis, lo que se denomina como Autosimilitud ${ }^{1}$ (MANDELBROT, 1987; QUEZADA, 2005, 2006). Así, en la solución de problemas de localización espacial se evidenció que las estrategias de solución de los participantes son fractales y su fractalidad era característica e individual, corroborando que el patrón fractal de dicha estrategia inferencial resulta ser un patrón estable que identifica al sistema (individuo) del cual proviene, incluso a diferentes edades de la persona (LABRA et al., 2000). Para la educación, esto puede ser iluminador respecto a las modalidades de aprendizaje que cada alumno tiene, es decir, podría pensarse razonablemente que todos los alumnos tienen la capacidad de aprender, sin embargo, sus patrones de incorporación de información, sus ciclos atencionales o sus ritmos de insight, podrían ser únicos e individuales y mantenerse como un sello identitario a lo largo de su vida escolar. Asimismo, da pie para reforzar la idea de que no existiría una "llave maestra" que despierte de igual forma las habilidades de los estudiantes, sino que estrategias que se ajustan mejor a las características individuales de cada alumno.

Ahora, a nivel de grupo, también podemos argumentar que existe un aprendizaje e inteligencia social. Aquí ya no hablamos de una red de neuronas, sino que de una red de individuos. Sabemos que muchas decisiones, tal vez las más complejas, se toman en grupo, ya que existe evidencia que las decisiones grupales son mejores que las individuales para un mismo contexto. En efecto, Hastie y Kameda (2005) demuestran empíricamente que las decisiones tomadas por grupos son sistemáticamente más eficientes que aquellas tomadas individualmente. Aún más, es notorio que el gran avance de la ciencia se ha asociado importantemente a la interacción de los miembros de la comunidad científica, y no por trabajos aislados. Un fenómeno similar se da en una organización, ya que muchos grupos de trabajo colaboran para lograr un objetivo, que sería imposible

1 Un ejemplo gráfico que podría clarificar una figura fractal en la naturaleza, se encuentra cuando se analiza la forma de una coliflor, en la cual un brazo de ella es una expresión en menor escala de la coliflor entera. Asimismo, se podría seguir seccionando la coliflor y se podría seguir encontrando que la forma en menor escala se sigue pareciendo a la mayor. Esto ejemplifica la característica de Autosimilitud, en la cual una parte se parece al todo o bien el todo es una magnificación de una parte (invariancia escalar). 
de alcanzar individualmente. Véase que no solamente el logro del objetivo es grupal, sino que como ya se dijo, el objetivo mismo es una manifestación de la interacción entre los diferentes agentes, sean estos individuos o grupos (COHEN et al., 1972). Podemos ver que las propiedades emergentes se producen a diferentes escalas y niveles de análisis; en este caso, a nivel neuronal, individual, grupal y organizacional. Un fenómeno emergente a cierto nivel sienta las bases para crear otro en un nivel superior y así sucesivamente.

En el caso del profesor y su curso, tenemos la misma situación: aprendizaje como fenómeno emergente a nivel neuronal, luego a nivel individual y a nivel de posibles grupos que se formen y finalmente a nivel del curso. De hecho, puede que los alumnos de buen rendimiento tiendan a agruparse para trabajar, lo mismo que los estudiantes de resultados más modestos. Esto tenderá a exacerbar las direcciones de la realimentación positiva. Por ello el profesor, consciente de que el aprendizaje es un fenómeno al menos en parte explicado por la interacción entre alumnos, puede pensar que una posible intervención sea forzar a que los alumnos de buen rendimiento trabajen con los de menor rendimiento para evitar la polarización del curso y mejorar el rendimiento global de éste. Tal vez el profesor propicie el trabajo en proyectos en grupo y designe a los integrantes de cada grupo, mezclando alumnos de buen y mal rendimiento. Sin embargo, eso puede empeorar la situación: los buenos alumnos podrían resentir tener que trabajar más para compensar por los alumnos de menor rendimiento y estos últimos puede que simplemente vean la oportunidad para trabajar aún menos de lo que lo hubieran hecho si hubiesen realizado el proyecto solos. Puede que se consiga nivelar las evaluaciones de los alumnos, pero no se logre el objetivo real: que los alumnos aprendan bien los contenidos del curso.

El fracaso de dicha intervención podría asociarse a que el profesor no reconoce estar en presencia de una propiedad emergente. Es cierto que el profesor entiende que el aprendizaje se crea en buena medida a partir de la interacción entre los alumnos y por eso trata de intervenir a nivel macro. Sin embargo, dicha interacción se basa en las reglas de comportamiento individual de cada agente, o sea, ocurre a nivel micro. Por ello, debemos analizar cómo esas reglas influirán en la interacción entre agentes y qué tipo de propiedad emergente crearán. Aquí radica justamente el problema con los fenómenos emergentes: se puede reconocerlos e identificar sus características solamente una vez que estos ocurren. El problema es que ese análisis ex-post nos dice poco respecto a cómo debemos intervenir a los agentes para que emerja el comportamiento que deseamos. En el ejemplo de la bandada, si se desea que ésta vuele en cierta dirección, no se le puede decir a cada pájaro que tome esa dirección, ya que estos mantienen solamente una posición relativa respecto a los otros, construyendo colectivamente una trayectoria de vuelo que resulta una propiedad emergente 
de la agregación de los comportamientos individuales. Por ello, en principio, se debería intervenir las reglas de comportamiento individual. Sin embargo, la pregunta es cómo se debe alterar dichas reglas para lograr que la bandada vuele en cierta dirección. Lo mismo sucede en nuestro ejemplo con los alumnos: el profesor debería diseñar la intervención para que los alumnos cooperen entre si y aprendan, pero eso es una propiedad emergente. Luego, se puede pensar que el profesor debe diseñar reglas que incentiven a que los buenos alumnos ayuden a los de menor rendimiento y, a su vez, que estos acepten trabajar. Sin embargo, la consecuencia de intervenir las reglas de comportamiento individual de los alumnos para que se alineen con dicho objetivo, no se sabrá hasta que se haya efectuado la acción y observado los resultados obtenidos en el sistema.

Por otra parte, dadas las propiedades caóticas de los SC, una muy pequeña variación en las condiciones iniciales del mismo, puede resultar en dinámicas completamente diferentes. Por decirlo de otra forma, las diferencias en las condiciones iniciales de un sistema se amplifican en el tiempo, no pudiéndose predecir el comportamiento del sistema en el futuro. Por ello, si tenemos buenos resultados con una intervención, puede que en una situación similar el aplicar la misma intervención no de buenos resultados. Dado esto, el paradigma de SC nos dice que la predictibilidad completa es inalcanzable y que el sistema se autoorganiza. Por otra parte, aunque en un sistema complejo no se sabe exactamente cómo una intervención modificará el comportamiento del mismo, tampoco se puede afirmar que éste sea aleatorio. Es decir, en SC no se puede lograr una predictibilidad exacta pero en caso alguno es un sistema azaroso, del cual no se puede describir regularidades. Es por ello, que existen algunos métodos y herramientas que nos permiten lidiar con dichas características de los SC.

Sabiendo que los SC exhiben propiedades emergentes y que la forma de intervenir a los agentes para crear propiedades que nos interesan es difícil de establecer ex-ante, un método que parece razonable es poder experimentar con un modelo del sistema. Con ello, se podrá diseñar intervenciones a nivel de los agentes y analizar con razonable validez los resultados que provocarán dichas intervenciones. Este método se conoce con el nombre de Modelamiento Basado en Agentes (MbA) o ABM (Agent-Based Modeling, por su sigla en inglés). Para explicar el MbA debemos reconocer que en el modelamiento de SC hay dos grandes tradiciones no excluyentes: Autómatas Celulares y Agentes (GILBERT; TROITZSCH, 1999). Los autómatas son celdas de un tablero que tienen un conjunto finito de valores posibles de estados que son modificados por la interacción con autómatas vecinos según leyes definidas por el programador (BERLEKAMP; CONWAY; GUY, 1982; VALLACHER; NOWAK, 1997). A esta línea de investigación adscriben las experiencias realizadas en ciencias sociales por el sociólogo Thomas C. Schelling (1978/1989) sobre dinámicas de 
segregación racial, la cual ya fue mencionada. Similarmente, los agentes pueden tener estados en los que se incluyen variadas características más elaboradas, tales como preferencias, creencias, memoria de eventos recientes y conexiones sociales (CANESSA; RIOLO, 2006). Cabe aclarar que un agente sería un sofisticado autómata celular o, éste un rudimentario agente. Variados ejemplos de aplicaciones de agentes al comportamiento organizacional se encuentran en Canessa y Riolo (2003), Zickar (2000), Stasser (2000), So y Durfee (1998), Huberman y Glance (1998), y Scacchi (1998).

En MbA un sistema se modela a partir del comportamiento individual de los agentes, usando una plataforma y software computacional. La bondad del MbA es su plasticidad y versatilidad para representar diversos tipos de comportamientos, ya sean humanos o de naturaleza inerte. Las ventajas principales son:

a) Proporciona una herramienta para modelar SC que no pueden describirse por otros métodos. Por ejemplo, en dinámica no lineal se usa frecuentemente ecuaciones diferenciales (STROGATZ, 1994), pero existen SC que incluso con ellas, no pueden ser representados.

b) Permite un adecuado modelamiento de la dinámica de SC, pudiendo comprender mejor su funcionamiento. Por ejemplo, el modelo de segregación social de Schelling ayuda a entender cómo se segrega un conjunto de personas, basado en interacciones entre agentes muy simples.

c) Aunque el MbA no puede predecir exactamente el comportamiento del sistema, ayuda a conocer su dinámica y tomar decisiones respecto a ella. En el modelo mencionado, entender la segregación permite diseñar y probar diferentes estrategias que disminuyan la probabilidad de que ocurra. En concreto, dentro del mundo educativo, podría ser un modelo que explique y anticipe problemas de convivencia escolar, bullying y, como ya se mencionó, segregación entre alumnos de distinto rendimiento.

d) El MbA ayuda a definir las variables más centrales que están implicadas en el comportamiento del sistema, sin realizar costosos experimentos, traduciéndose en economía de recursos. Incluso, en ocasiones los experimentos en el sistema real no podrían realizarse debido a, por ejemplo, limitaciones prácticas o éticas, que impliquen someter a personas a tratos vejatorios. Esto no significa que $\mathrm{MbA}$ reemplaza a la experimentación en el sistema real, sino que la asiste y la complementa. 
e) $\mathrm{MbA}$ facilita la fertilización cruzada entre las ciencias naturales y ciencias sociales, ya que facilita la expresión algorítmica de teorías del comportamiento en un lenguaje afín. Esta interdisciplinariedad es la que alentó Piaget (1979) y que ha sido un llamado constante en la investigación en SC, particularmente en relación al estudio de los procesos mentales (ALBUS et al., 2007). Por esta razón, pareciera provechoso para las ciencias de la educación participar de esta interdisciplinariedad que se está dando entre las ciencias sociales y naturales, bajo el alero del paradigma de sistemas complejos.

Recapitulando, se puede decir que el uso de MbA consiste en reproducir el comportamiento de un SC y luego experimentar con dicho modelo para obtener conclusiones suficientemente adecuadas al propósito del análisis. Con esto podemos lidiar con las propiedades emergentes al tener una herramienta que nos permita analizar el comportamiento de un $\mathrm{SC}$, ver preliminarmente cuáles podrían ser las intervenciones útiles para lograr el comportamiento deseado del SC y probarlas en dicho modelo para refinarlas iterativamente. Una vez que se obtenga suficiente evidencia que las intervenciones logran los resultados requeridos, se pueden implementar en el sistema real. Además, el poder experimentar con el MbA nos permite ver qué tan sensible a condiciones iniciales es el sistema, con lo cual podremos comprobar mejor las probabilidades de éxito que tendremos con las intervenciones y qué tan generalizables a otras situaciones serían estas.

La perspectiva propuesta en este artículo brinda una nueva manera de comprender los fenómenos educativos, complementando la mirada tradicional. Lejos de pensar que la educación se ciña por fenómenos lineales, hemos presentado ejemplos que ponen de manifiesto que otros factores (no-lineales) podrían hacerse presentes. No obstante, este camino no es fácil, aunque puede verse auxiliado por la ayuda de herramientas tales como MbA (WILENSKY; RAND, 2007). En la educación, la aplicación de MbA requiere tanto experiencia en modelaje como conocimiento del fenómeno educativo. Esto último alienta el llamado al trabajo interdisciplinario entre investigadores en ciencias de la educación y en análisis de sistemas complejos. 


\section{Conclusiones y desafios}

Se ha visto que muchas veces, nuestra intuición basada en un modelo lineal de causa-efecto de un sistema, lleva a analizarlo y sacar conclusiones no válidas en relación con el comportamiento del mismo. Aún más, en base a dicho análisis se puede diseñar intervenciones que no contribuirán a lograr los objetivos que se persiguen. Para no correr dicho riesgo, es conveniente complementar dicha mirada con la perspectiva de los SC. Si bien es cierto, este paradigma no es el único posible de emplear, en muchas situaciones proporciona los métodos y herramientas que son los más adecuados para analizar mejor a un sistema e intervenirlo para lograr un objetivo. Indudablemente que esta perspectiva de SC es bastante más difícil de emplear y más onerosa que usar un paradigma lineal, pero muchas veces es la única forma de asegurar un resultado aceptable.

En efecto, comúnmente al pensar aplicando solamente el paradigma lineal, se puede caer en la trampa de centrarse en identificar un único foco de análisis e intervención en educación. Por un lado, se ha visto aquí que enfocarse en los desempeños individuales y modificarlos por acciones o intervenciones exclusivamente a nivel individual, podría no tener los resultados esperados. Por otro, focalizarse en la indesmentible importancia de las variables de contexto, y muy especialmente aquellas vinculadas con el grupo más amplio, tampoco explica completamente el éxito o fracaso en un rendimiento individual. Por lo tanto, al hablar en sistemas complejos, atendemos a la íntima relación (acaso diálogo) entre los niveles micro y macro, proponiendo intervenciones en ambos niveles, pero sin pretender resultados exactos y lineales, sino que propuestas que tengan una mayor probabilidad de ocurrencia.

Por otra parte, dadas las propiedades caóticas de un SC, o sea, su sensibilidad a condiciones iniciales, la utilización de este paradigma también permite alertar al investigador respecto a la aplicabilidad de ciertas intervenciones a un sistema, dentro de un contexto particular, a otros contextos y/o situaciones. Es decir, debe tenerse mucho cuidado al establecer la generalización de intervenciones.

Todo lo visto en relación al paradigma de SC y al mejor entendimiento que se logra del fenómeno de aprendizaje al aplicarlo, sería inútil si no se tuviera un método para ponerlo en práctica. Justamente el MbA permite operacionalizar la aplicación del paradigma de SC. Usando MbA se puede modelar un SC, y luego analizarlo para extraer sus características más relevantes, de acuerdo al objetivo que se persiga. Entonces, se puede experimentar con dicho modelo para encontrar intervenciones adecuadas. No decimos que realizar todo lo anterior con $\mathrm{MbA}$ 
sea fácil, pero al menos se tiene una herramienta que permite hacerlo.

Ahora, abordando específicamente el fenómeno educacional, si se considera los procesos de aula como expresiones de comportamiento complejo, es esperable también que distintas intervenciones tengan resultados completamente diferentes, originados en la sensibilidad que tienen los sistemas y, muy particularmente, aquellos compuestos por seres humanos. Un ejemplo cotidiano se puede observar intuitivamente cuando un profesor realiza una misma clase en cursos distintos, realizando pequeñas (a veces involuntarias) modificaciones que posteriormente obtiene distintas consecuencias en diversos ámbitos: rendimiento, atención, motivación, simpatía, etc. Por lo tanto, el desafío es descubrir qué intervenciones tendrán una mayor probabilidad de éxito en función de los objetivos planteados por el proyecto educativo.

Este artículo se ha enfocado en ilustrar lo que sucede dentro de las paredes de un aula, donde se desarrolla una serie de procesos de gran complejidad, los cuales podrían analizarse adecuadamente bajo la perspectiva de SC. Sin embargo, no es menos cierto que los procesos educativos no sólo se limitan al salón de clases, sino que se extienden mucho más allá de sus límites físicos. Tan sólo como ejemplo, al analizar la motivación de un alumno, se ve que tiene una expresión individual, pero que también depende de la motivación que el profesor pueda promover, del involucramiento de los padres o tutores del alumno $\mathrm{o}$, incluso, de la importancia que la educación tenga para la comunidad en la que el alumno está inmerso. No obstante, tampoco sería apropiado sumar más y más variables, sino sólo aquellas que tengan una real importancia para modelar adecuadamente el fenómeno, según los objetivos del análisis.

Finalmente, a lo largo de este artículo se ha pretendido evidenciar que los procesos implicados en la educación podrían analizarse a través de una nueva mirada de distintos fenómenos de la naturaleza a través de un nuevo marco ligado a los Sistemas Complejos. La inclusión de esta nueva mirada no obedece a una curiosidad o mera erudición, sino que responde precisamente a la necesidad de explicar una serie de fenómenos, que las perspectivas epistemológicas previas dejaban fuera o comprendían sólo parcialmente. Los Sistemas Complejos no sólo explican mejor sino que pueden dar pistas para diseñar intervenciones educativas más certeras. Si los procesos educativos corresponden a fenómenos complejos, esto no hará necesariamente más difícil la intervención o reducirá las expectativas de éxito, pero reconocer eso permitirá que dicha intervención sea menos ingenua y pondrá límites realistas a los resultados esperados. Este marco permite abrazar la complejidad inherente al fenómeno y, así, promover las acciones más adecuadas para los fines del proyecto educativo. 


\section{REFERENCIAS}

ALBUS, J. A.; BEKEY, G. A.; HOLLAND, J. H.; KANWISHER, N. G.; KRICHMAR, J. L.; MUSHKIN, M.; MODHA, D. S.; RAICHLE, M. E.; SHEPHERD, G. M.; TONONI, G. A. Proposal for a decade of the mind initiative. Science, 317, p. 1321-1322, 2007.

BERLEKAMP, E.; CONWAY, J.; GUY, R. Winning ways for your mathematical plays, games in particular, 2, London: Academic Press, 1982.

CANESSA, E.; RIOLO, R. The effect of organizational communication media on organizational culture and performance: an agent-based simulation model. Computational and Mathematical Organization Theory, v. 9. n.2, p. 147-176, 2003.

CANESSA, E.; RIOLO, R.. An agent-based model of the impact of computer-mediated communication on organizational culture and performance: an example of the application of complex systems analysis tools to the study of CIS. Journal of Information Technology, v. 21, p. 272-283, 2006.

COHEN, M. D.; MARCH, J. G.; OLSEN, J. P. A garbage can model of organizational choice. Administrative Science Quarterly, v. 17, n. 1, p. 1-25, 1972.

GILBERT, N.; TROITZSCH, K. Simulation for the social scientist. Buckingham, England: Open University Press, 1999.

GLAUBER, R. J. Time-dependent statistics of the Ising model. Journal of Mathematical Physics, v. 4, p. 294, 1963.

HASTIE, R.; KAMEDA, T. The robust beauty of majority rules in group decisions. Psychological Review, v. 112, p. 494-508, 2005.

HOLLAND, J. H.; MILLER, J. H. Learning and adaptive economic behavior. American Economic Review, v. 81, n. 2, p. 365-370, 1991.

HUBERMAN, B. Y.; GLANCE, N. Fluctuating efforts and sustainable cooperation. In: PRIETULA, M. J.; CARLEY, K. M.; GASSER, L. (Eds.). Simulating organizations. Cambridge MA: The MIT Press, 1998.

LABRA, F.; QUEZADA, A.; CAÑETE, O.; BASAURE, M.; MORA, P. Análisis geométrico de la dinámica inferencial: de la infancia a la adolescencia. Revista de Psicología de la Universidad de Chile, IX, 2000.

MANDELBROT, B. Los objetos fractales: forma, azar y dimensión. Barcelona: Tus- 
quets (Orig. 1977), 1987.

NICOLIS, G.; NICOLIS, C. Foundations of complex systems: nonlinear dynamic statistical physics information and prediction. Singapore: World Scientific Publishing Company, 2007.

PIAGET, J. Relations between psychology and other sciences. Annual Review of Psychology, v. 30, n. 1, p. 1-8, 1979.

QUEZADA, A.. Fractales en el estudio de la Psicología. Revista Digital Universitaria, v. 7, n. 10, p. 1-12, 2006.

QUEZADA, A. Fractales, más allá de 1D, 2D o 3 D. Revista Digital Universitaria, v. 6, n. 12, p. 1-14, 2005.

RUMELHART, D. E.; MCCLELLAND, J. L., and the PDP research group. Parallel distributed processing: Explorations in the microstructure of cognition. Volume I. Cambridge, MA: MIT Press, 1986.

SHANNON, C. E. A Mathematical Theory of Communication. Bell System Technical Journal, v. 27, p. 379-423, 1948.

SCHELLING, T. Micromotivos y macroconducta. México, D. F.: Fondo de Cultura Económica, S. A. de C. V. (Orig. 1978), 1989.

SCACCHI, W. Modeling, simulating, and enacting complex organizational processes: a life cycle approach. In: PRIETULA, M. J.; CARLEY, K. M.; GASSER, Y. L. (Eds.). Simulating organizations. Cambridge MA: The MIT Press, 1998.

SO, Y.; DURFEE, E. H. Designing organizations for computational agents. In: PRIETULA, M. J.; CARLEY, K. M.; GASSER, Y. L. (Eds.). Simulating organizations. Cambridge MA: The MIT Press, 1998.

SOLÉ, R. V.; MANRUBIA, S. Orden y caos en sistemas complejos. Fundamentos. Barcelona: Edicions UPC, 2001.

STASSER, G. Information distribution, participation, and group decision: Exploration with the DISCUSS and SPEAK models. In: ILGEN, D. R.; HULIN, CH. L. (Eds.). Computational modeling of behavior in organizations. Washington D.C.: American Psychological Association, 2000.

STROGATZ, S. Nonlinear dynamics and chaos. New York: Perseus Books Publishing, 1994.

VALLACHER, R. R.; NOWAK, A. The emergence of dynamical social psychology. 
Psychological Inquiry, v. 8, n. 2, p. 73-99, 1997.

WILENSKY, U.; Y RAND, W. Making models match: Replicating agent-based models. Journal of Artificial Societies and Social Simulation, v. 10, p. 42, 2007.

ZICKAR, M. Modeling faking on personality tests. In: ILGEN, D. R.; HULIN, CH. L. (Eds.). Computational modeling of behavior in organizations. Washington D.C.: American Psychological Association, 2000.

Texto recebido em 04 de maio de 2008. Texto aprovado em 30 de junho de 2008 . 